PROCEEDINGS OF THE

AMERICAN MATHEMATICAL SOCIETY

Volume 132, Number 4, Pages 1223-1228

S 0002-9939(03)07168-5

Article electronically published on August 20, 2003

\title{
BUNDLES WITH PERIODIC MAPS AND MOD $p$ CHERN POLYNOMIAL
}

\author{
JAN JAWOROWSKI
}

(Communicated by Paul Goerss)

\begin{abstract}
Suppose that $E \rightarrow B$ is a vector bundle with a linear periodic map of period $p$; the map is assumed free on the outside of the 0 -section. A polynomial $c_{E}(y)$, called a mod $p$ Chern polynomial of $E$, is defined. It is analogous to the Stiefel-Whitney polynomial defined by Dold for real vector bundles with the antipodal involution. The $\bmod p$ Chern polynomial can be used to measure the size of the periodic coincidence set for fibre preserving maps of the unit sphere bundle of $E$ into another vector bundle.
\end{abstract}

1. Introduction. In 2] Albrecht Dold defined the Stiefel-Whitney polynomials for vector bundles with the antipodal involution; it is a useful tool in studying "parametrized" Borsuk-Ulam type problems (compare also [4]). Given two vector bundles $E \rightarrow B$ and $E^{\prime} \rightarrow B$, with $S(E) \rightarrow B$ being the unit sphere bundle of $E$, and a fibre preserving equivariant map $f: S(E) \rightarrow E^{\prime}$, Dold used the StiefelWhitney polynomials to estimate the size of the zero set $Z(f)=f^{-1} 0$ of $f$, where 0 is the zero section of $E$. Since $f$ is equivariant, the zero set of $f$ is the set of antipodal coincidences of $f$, i.e., the set $A(f)=\{x \in S(E) \mid f x=f(-x)\}$.

In this paper we make similar constructions for vector bundles with linear periodic fibre preserving maps of a prime period $p$ that are free on the outside of the 0 -section. Given such a bundle $E \rightarrow B$ over a base space $B$ (which is assumed to be a CW-complex), we define a mod $p$ Chern polynomial, $c_{E}(y)$, associated to $E$. Using the Chern polynomial, we obtain a lower bound on the size of the periodic coincidence set $A(f)$ for fibre preserving maps $f: S(E) \rightarrow E^{\prime}$ of the unit sphere bundle of $E$ into another vector bundle in terms of the (cohomology) dimension. The periodic coincidence set $A(f)$ is the set of points $x \in S(E)$ such that $f$ maps the entire orbit of $x$ to a single point.

In Section 7 we prove our result for equivariant maps $E \rightarrow W$, where $W$ is another vector bundle over $B$ with a linear periodic fibre preserving map of a prime period, free on the outside of the 0 -section. In this case, $A(f)=Z(f)$. In Section 8 we give an estimation of the size of the periodic coincidence set for maps that are not necessarily equivariant; this requires some additional constructions.

Throughout the paper we will use cohomology of the Čech type. The Čech cohomology has a continuity property, which says that if a cohomology class vanishes on

Received by the editors August 7, 2002 and, in revised form, November 22, 2002.

2000 Mathematics Subject Classification. Primary 55R91, 55R40; Secondary 55M20.

Key words and phrases. Periodic map, fibre preserving map, complex structure, Chern classes, lens space, Chern polynomial, coincidence set. 
a closed set, then it vanishes on a neighborhood of this set. Throughout the paper (with the exception of the example in Section 2 below), we will use the cohomology with coefficients in $\mathbf{Z}_{p}$, and the coefficient group will be suppressed in the notation: $H^{*}()=H^{*}\left(; \mathbf{Z}_{p}\right)$.

If $G$ is a topological group, $B G$ is a classyfying space for $G, Y$ is a space, and $u \in H^{*}(B G)$, we denote by $u \mid Y$ the image of $u$ in $H^{*} Y$ under the homomorphism induced by a classifying map $Y \rightarrow B G$ ("the restriction" of $u$ to $Y$ ).

If $X$ is a $G$-space, we denote by $\bar{X}=X / G$ the orbit space of the action.

2. Some examples. The problem of extending Borsuk-Ulam type theorems from a single sphere to a bundle of spheres is nontrivial even for trivial bundles as the following example shows (in this case $p=2$ ).

Example. Let $f: S^{1} \times S^{1} \rightarrow \mathbf{R}$ be a real-valued function. Then the coincidence set $A(f)=\left\{(x, y) \in S^{1} \times S^{1} \mid f(x, y)=f(-x, y)\right\}$ and $H^{1}\left(A(f) ; \mathbf{Z}_{2}\right)$ has $H^{1}\left(S^{1} ; \mathbf{Z}_{2}\right) \cong$ $\mathbf{Z}_{2}$ as a direct summand.

This is a consequence of Theorem 1 of [4. This fact is related to a result by J. E. Connett [1], which was a motivation for [4].

The parametrized Borsuk-Ulam theorems (in the case of $p=2$ or in the complex case, for $G=S^{1}$ ) can be applied to estimate the "size" (in terms of the cohomological dimension or the topological dimension) of the kernel of a linear vector preserving map from one vector bundle into another; compare [3] and [2, p. 282.

3. Linear periodic maps. Suppose that $V$ is a finite-dimensional real vector space with a scalar product, and let $\tau: V \rightarrow V$ be an orthogonal periodic map of a period $p>2$ without fixed points other than 0 . Then the dimension of $V$ must be even, and we can use $p$ to define a complex structure on $V$ as follows.

We first construct an orthogonal map $\sigma: V \rightarrow V$ of period $4 p$ (a fourth root of $\tau$ ). Given a vector $v \in V$, the vectors $v$ and $\tau v$ are never opposite for $p>2$. They determine a two-dimensional subspace $P$ (a plane) in $V$ and a unit circle in it. They also determine an orientation in $P$ along the smaller of the two arcs of the circle from $v$ to $\tau v$. For any other vector $w$ on that circle, let $b(v, w)$ be the unit vector bisecting the oriented pair $v, w$. Let $\sigma(v)=b(v, b(v, \tau(v)))$, so that $\sigma^{4}=\tau$. Now we can set $i=\sigma^{p}$. Then $i$ is linear and $i^{2}=-1$; so $i$ is a complex strtucture.

The map $\tau$ becomes a unitary map and can be diagonalized. The matrix of $\tau$ is a diagonal matrix whose entries are $p$-th roots of unity other than 1 .

If $\operatorname{dim} V=2 n+2$ and $S^{2 n+1}$ is the unit sphere in $V$, then the orbit space $S^{2 n+1} / \mathbf{Z}_{p}$ of the $\mathbf{Z}_{p}$-action generated by $\tau$ is a lens space $L_{p}^{2 n+1}$.

4. Comments on the classifying space for $\mathbf{Z}_{p}$. From now on we assume that $p$ is a prime number greater than 2 .

We would like to look closer at the cohomology of the classifying space for $\mathbf{Z}_{p}$ and compare it with the cohomology of $B S^{1}$.

A model for the classifying bundle for $\mathbf{Z}_{p}$ is the $p$-fold covering $\alpha: S^{\infty} \rightarrow B \mathbf{Z}_{p}=$ $L_{p}^{\infty}$, where $S^{\infty}$ is the unit sphere in the infinite-dimensional complex vector space $\mathbf{C}^{\infty}, \alpha$ is the orbit map of the standard action, multiplication by $\exp \left(\frac{2 \pi \sqrt{-1}}{p}\right)$, and the orbit space, $L_{p}^{\infty}$, is an infinite-dimensional lens space.

A model for the classifying bundle for $S^{1}$ is the Hopf bundle $\beta: S^{\infty} \rightarrow P_{\infty} \mathbf{C}$ over the infinite-dimensional complex projective space $P^{\infty} \mathbf{C}$. We have a commutative 
diagram

$$
\begin{array}{ccc}
S^{\infty} \quad \stackrel{\alpha}{\longrightarrow} & L_{p}^{\infty} \\
& & \downarrow \pi \\
& P_{\infty} \mathbf{C}
\end{array}
$$

It is well known that $H^{*}\left(L_{p}^{\infty}\right) \cong \Lambda[b] \otimes \mathbf{Z}_{p}[c]$, where $b \in H^{1}\left(L_{p}^{\infty}\right)$ and $c \in H^{2}\left(L_{p}^{\infty}\right)$ is the $\bmod p$ reduction of the first Chern class. By comparing the Gysin sequences of the $S^{1}$-bundles $\alpha$ and $\beta$ we see that the map

$$
H^{*}\left(P_{\infty} \mathbf{C}\right) \cong \mathbf{Z}_{p}[c] \stackrel{\pi^{*}}{\longrightarrow} \Lambda[b] \otimes \mathbf{Z}_{p}[c] \cong H^{*}\left(L_{p}^{\infty}\right)
$$

is injective in even dimensions.

We will refer to $b$ and $c$ as the universal characteristic classes of $\mathbf{Z}_{p}$.

5. $\mathbf{Z}_{p}$-bundles. Suppose that $E \rightarrow B$ is a real vector bundle over $B$, with a Riemannian metric, of a real fibre dimension $2 n+2$ and with a fibre preserving orthogonal action of $\mathbf{Z}_{p}$ that is free outside the zero section. By comments of Section 3, $E \rightarrow B$ can be given a complex structure so that the action of $\mathbf{Z}_{p}$ is unitary (compare [6], p. 149).

Let $S(E) \rightarrow B$ be the unit sphere bundle of $E$; its fibre is $S^{2 n+1}$. Let $P(E) \rightarrow B$ be the (complex) projective bundle of $S(E) \rightarrow B$ and let $L(E) \rightarrow B$ be the lens space bundle, arising from the standard $\mathbf{Z}_{p}$-action, associated to $E$. The fibre of $P(E)$ is the complex projective space $P_{n} \mathbf{C}$, and the fibre of $L(E)$ is the lens space $L_{p}^{2 n+1} . L(E)$ admits a free fibre preserving action of $S^{1}$.

The $H^{*}(B)$-modules $H^{*}(P(E))$ and $H^{*}(L(E))$ admit Leray-Hirsch bases. A basis for $H^{*}(P(E))$ is $\left\{1, u, \ldots, u^{n}\right\}$, where $u=c \mid P(E)$, the image of the first universal Chern class $c=c_{1} \in H^{2}\left(P_{\infty} \mathbf{C}\right)$ under the homomorphism induced by a classifying map $P(E) \rightarrow P_{\infty}$ C. A Leray-Hirsch basis for $H^{*}(L(E))$ is

$$
\left\{1, s, t, s t, t^{2}, \ldots, s t^{n}\right\},
$$

where $s=b \mid L(E)$ and $t=c \mid L(E)$ are the images of the universal classes $b$ and $c$ of $\mathbf{Z}_{p}$ under the homomorphism induced by a classifying map $L(E) \rightarrow L_{p}^{\infty}$.

Proposition 1. $L(E) / S^{1} \cong P(E)$, and the orbit map $\rho: L(E) \rightarrow L(E) / S^{1}=$ $P(E)$ induces a monomorphism $\rho^{*}: H^{*}(P(E)) \rightarrow H^{*}(L(E))$ of $H^{*}(B)$-algebras that is zero in odd dimensions and is an isomorphism of the even-dimensional subalgebras.

Proof. This is a consequence of Propositions 1 and the Leray-Hirsch theorem ([7], p. 365).

In particular, $t^{n+1}$ can be written as a linear combination

$$
t^{n+1}=\sum_{i=1}^{n+1}(-1)^{i} c_{i} t^{n-i+1}
$$

where $c_{i} \in H^{*}(B)$ is the mod $p$ Chern class of the bundle $E \rightarrow B$ reduced mod $p$ (compare [7], p. 377). 
6. Mod $p$ Chern polynomial. Consider the algebra $\Lambda[x] \otimes H^{*}(B)[y]$ over $H^{*}(B)$.

Definition. Suppose that $E \rightarrow B$ is a bundle as in in Section 5 of a (real) fibre dimension $2 n+2$, with an orthogonal fibre preserving periodic map of period $p$. The $\bmod p$ Chern polynomial of $E$ is

$$
c_{E}(y)=\sum_{i=0}^{n+1}(-1)^{i} c_{i} y^{n+1-i} \in \Lambda[x] \otimes H^{*}(B)[y] .
$$

The map

$$
\begin{gathered}
e_{E}: \Lambda[x] \otimes\left(H^{*} B\right)[y] \rightarrow H^{*}(L(E)), \\
x \mapsto b|L(E)=s, y \mapsto c| L(E)=t
\end{gathered}
$$

will be called the evaluation map; it is a homomorphism of $H^{*}(B)$-algebras.

Proposition 2. Ker $e_{E}=\left(c_{E}(y)\right)$, the ideal in $\Lambda[x] \otimes\left(H^{*}(B)[y]\right.$ generated by the Chern polynomial $c_{E}(y)$.

Proof. First, $c_{E}(y) \mid L(E)=0$, by the definition.

Now let $p(x, y)$ be a polynomial in $\Lambda[x] \otimes H^{*}(B)[y]$ such that $p(x, y) \mid L(E)=0$. By dividing $c_{E}(y)$ into $p(x, y)$ we can write $p(x, y)$ as $p(x, y)=q(x, y) \cdot c_{E}(y)+r(x, y)$ where $\operatorname{deg}(r(x, y))<2 n+2$. Since $c_{E}(t)=0$ and the set $\left\{1, s, t, s t, \ldots, s t^{n}\right\}$ is linearly independent, $r(x, y)=0$.

7. The zero set for equivariant maps. Suppose that $E \rightarrow B$ is a real vector bundle over $B$ of a real fibre dimension $2 n+2$ with a fibre preserving orthogonal action of $\mathbf{Z}_{p}$, free outside the zero section, as in Section 5. Let $S(E)$ be the unit sphere bundle of $E$, let $W \rightarrow B$ be another real vector bundle over $B$, also with a fibrewise action of $\mathbf{Z}_{p}$, free outside the zero section (so that the fibre dimension of $W$ must be even). Let $g: S(E) \rightarrow W$ be an equivariant fibre preserving map.

The zero set $Z(g)=\{x \in S(E) \mid g x=0\}$ is an equivariant subset of $S(E)$.

Proposition 3. If a polynomial $p(x, y) \in \Lambda[x] \otimes H^{*}(B)[y]$ vanishes on $\overline{Z(g)}$, then there exists a polynomial $q(x, y) \in \Lambda[x] \otimes H^{*}(B)[y]$ such that

$$
p(x, y) \cdot c_{W}(y)=q(x, y) \cdot c_{E}(y) .
$$

In terms of ideals, if $e_{Z(g)}: \Lambda[x] \otimes H^{*}(B)[y] \rightarrow H^{*}(\overline{Z(g)})$ is the evaluation map for $Z(g)$, then

$$
\operatorname{Ker}\left(e_{Z(g)}\right) \cdot\left(c_{W}(y)\right) \subset\left(c_{E}(y)\right) .
$$

Proof. Suppose that $p(x, y)$ vanishes on $\overline{Z(g)}$. Then, by the continuity of Čech cohomology, there is an invariant neighborhood $N$ of $Z(g)$ in $S(E)$ such that $p(x, y) \mid \bar{N}=$ 0 . By the exactness of the cohomology sequence of the pair $(L(E), \bar{N})$, there is a $u \in H^{*}(L(E), \bar{N})$ such that $p(x, y) \mid L(E)=i^{*} u$, where $i: L(E) \rightarrow(L(E), \bar{N})$ is the inclusion.

On the other hand, the map $g_{0}: S(E)-Z(g) \rightarrow W-0$ induced by $g$ is an equivariant map and an $H^{*}(B)$-algebra homomorphism. If $\overline{g_{0}}$ is the map of the orbit spaces induced by $g_{0}$, then $c_{W}(y) \mid(L(E)-\overline{Z(g)})=\bar{g}_{0}^{*}\left(c_{W}(y) \mid(\overline{W-0})\right)$, since $\bar{g}_{0}^{*}$ is a homomorphism of $H^{*}(B)$-algebras. But $W-0$ is fibrewise equivariantly homotopically equivalent to the sphere bundle $S(W)$ of $W$; so $\overline{W-0} \simeq L(W)$, and hence $c_{W}(y) \mid(\overline{W-0})=0$. By exactness of the cohomology sequence of $(L(E), L(E)-\overline{Z(g)})$, there is a $v \in H^{*}(L(E), L(E)-\overline{Z(g)})$ such that $i^{*} v=$ $c_{W}(y) \mid L(E)$. But then $\left.u \cdot v \in H^{*}(L(E), \bar{N} \cup(L(E)-\overline{Z(g)}))\right)=H^{*}(L(E), L(E))=0$. 
So $\left(p(x, y) \cdot c_{W}(y)\right) \mid L(E)=0$. By Proposition $5, p(x, y) \cdot c_{W}(y)$ must be a multiple of $c_{E}(y)$.

8. Estimating the size of the coincidence set. Suppose that $E \rightarrow B$ is a real vector bundle over $B$ of a real fibre dimension $2 n+2$ with a fibre preserving orthogonal action of $\mathbf{Z}_{p}$, free outside the zero section, as in Section 5. Suppose that $\tau: E \rightarrow E$ is a generator of the action. Let $S(E)$ be the unit sphere bundle of $E$, let $F \rightarrow B$ be another real vector bundle over $B$ of fibre dimension $k$ and let $f: S(E) \rightarrow F$ be a fibre preserving map. We do not assume that $F$ has a $\mathbf{Z}_{p}$-action, or, even if it has one, that $f$ is equivariant.

Definition. The periodic coincidence set $A(f)$ is the set of points $x \in S(E)$ such that $f$ maps the entire $\mathbf{Z}_{p}$-orbit of $x$ to a single point.

$A(f)$ is an equivariant subset of $S(E)$.

To estimate the size of $A(f)$ we will first "symmetrize" $f$ to have an equivariant fibre preserving map from $E$ to another $\mathbf{Z}_{p}$-bundle.

Let $V=p \cdot F=F \oplus \cdots \oplus F$ ( $p$ times). Then $\mathbf{Z}_{p}$ acts on $V$ by cyclically permuting the coordinates, and the fixed point set of the action is the diagonal $D$ in $V$; it is a subbundle of $V$. Let $D^{\perp}$ be the orthogonal complement of $D$ in $V$. Then $D^{\perp}$ is an equivariant subbundle of $D$ and the action on $D^{\perp}$ is orthogonal and free on the outside of the zero section. The linear projection "along the diagonal" defines an equivariant fibre preserving map $r:(V, V-D) \rightarrow\left(D^{\perp}, D^{\perp}-0\right)$, where 0 is the zero section of $D^{\perp}$; it is a fibrewise equivariant homotopy equivalence.

The map $f$ induces an equivariant fibre preserving map $h: S(E) \rightarrow V$ by $z \mapsto\left(f z, f(\tau z), \ldots, f\left(\tau^{p-1} z\right)\right)$, with $h^{-1} D=A(f)$. The map $h$ followed by the projection $r$ induces an equivariant fibre preserving map $\left(g, g_{0}\right):(S(E), S(E)-$ $A(f)) \rightarrow\left(D^{\perp}, D^{\perp}-0\right)$. Moreover, $Z(g)=A(f)$.

The fibre dimension $D^{\perp}$ is $k(p-1)$, which is even. By the comments in Section $3, D^{\perp}$ admits a complex structure of a complex dimension $\frac{k(p-1)}{2}$ and hence a mod $p$ Chern polynomial, $c_{D^{\perp}}(y)$. Applying Proposition 4 to $W=c_{D^{\perp}}$ we obtain the following result.

Proposition 4. If a polynomial $p(x, y) \in \Lambda[x] \otimes H^{*}(B)[y]$ vanishes on $\overline{A(f)}$, then there exists a polynomial $q(x, y) \in \Lambda[x] \otimes H^{*}(B)[y]$ such that

$$
p(x, y) \cdot c_{D^{\perp}}(y)=q(x, y) \cdot c_{E}(y) .
$$

Again, in terms of ideals,

$$
\operatorname{Ker}\left(e_{A(f)}\right) \cdot\left(c_{D^{\perp}}(y)\right) \subset\left(c_{E}(y)\right) .
$$

The degree of $c_{E}(y)$ is $2 n+2$ and the degree of $c_{D^{\perp}}(y)$ is $k(p-1)$. Thus the evaluation map is nonzero for all polynomials whose degree is less than or equal to $2 n+1-k(p-1)$. This yields the following theorem.

\section{Theorem.}

$$
\begin{gathered}
\sum_{i=0}^{n-\frac{k(p-1)}{2}}\left(H^{*}(B)\right) y^{i} \oplus \sum_{i=0}^{n-\frac{k(p-1)}{2}}\left(H^{*}(B)\right) x y^{i} \stackrel{e_{A(f)}}{\longrightarrow} H^{*}(\overline{A(f)}), \\
x \mapsto s|\overline{A(f)}, \quad y \mapsto t| \overline{A(f)}
\end{gathered}
$$

is a monomorphism. 
In other words, for all polynomials $p(x, y)$ whose total degree in $x$ and $y$ is smaller than $2 n+2-k(p-1), p(x, y) \mid \overline{A(f)} \neq 0$.

In terms of the cohomology dimension, we have

Corollary. cohom.dim $\overline{A(f)} \geq$ cohom.dim $B+2 n+1-k(p-1)$.

Comment. The inequality in the Corollary is sharp, because it is sharp in the classical case of the Borsuk-Ulam theorem, for $B=$ point.

\section{REFERENCES}

1. J. E. Connett, On the cohomology of fixed-point sets and coincidence-point sets,, Indiana Univ. Math. J. 24 (1974-75), 627-634. MR 51:1805

2. A. Dold, Parametrized Borsuk-Ulam theorems, Comm. Math. Helv. 63 (1988), 275-285. MR 89h:55001

3. J. Jaworowski, Fibre preserving involutions and the kernel of the derivative (preprint), Forschungsinstitut fuer Mathematik ETH Zuerich, August, 1980.

4. J. Jaworowski, Fibre preserving maps of sphere bundles into vector space bundles, Lecture Notes in Mathematics 886, Springer-Verlag, New York, 1981, pp. 143-150. MR 83a:55002

5. J. Jaworowski, The Index of Free Circle Actions in Lens Spaces, Topology and its Appl. 123 (2002), 125-129. MR 2003g:57057

6. J. W. Milnor and J. D. Stasheff, Characteristic Classes, Annals of Math. Studies 76, Princeton University Press, Princeton, NJ, 1974. MR 55:13428

7. R. M. Switzer, Algebraic Topology - Homotopy and Homology, Grundlehren der mathematischen Wissenschaften, Band 212, Springer-Verlag, Berlin-Heidelberg-New York, 1975. MR 52:6695

Department of Mathematics, Indiana University, Bloomington, Indiana 47405-5701

E-mail address: jaworows@indiana.edu 